\title{
Stability of sequence-based control with random delays and dropouts
}

by Daniel E. Quevedo and Isabel Jurado

Copyright (C) 2014 IEEE.

This is an author-prepared version of the article, reprinted from IEEE Transactions on Automatic Control Vol. 59, Issue 5, p. 1296-1302 (2014)

http://dx.doi.org/10.1109/TAC.2013.2286911

This material is posted here with permission of the IEEE. Such permission of the IEEE does not in any way imply IEEE endorsement of any of University of Newcastle's products or services. Internal or personal use of this material is permitted. However, permission to reprint/republish this material for advertising or promotional purposes or for creating new collective works for resale or redistribution must be obtained from the IEEE by writing to pubs-permissions@ieee.org. By choosing to view this document, you agree to all provisions of the copyright laws protecting it. 


\title{
Stability of Sequence-Based Control with Random Delays and Dropouts
}

\author{
Daniel E. Quevedo, Member, IEEE, and Isabel Jurado
}

\begin{abstract}
We study networked control of non-linear systems where system states and tentative plant input sequences are transmitted over unreliable communication channels. The sequences are calculated recursively by using a pre-designed nominally stabilizing state-feedback control mapping to plant state predictions. The controller does not require receipt acknowledgments or knowledge of delay or dropout distributions. For the i.i.d. case, in which case the numbers of consecutive dropouts are geometrically distributed, we show how the resulting closed loop system can be modeled as a Markov non-linear jump system and establish sufficient conditions for stochastic stability.
\end{abstract}

\section{INTRODUCTION}

There exists significant interest in implementing closed loop control systems using wireless communication networks. Such Networked Control Systems (NCSs) are easier to install and to maintain than their traditional counterparts equipped with dedicated hardwired links. However, using off-the-shelf network technology for closedloop control gives rise to various challenges. In particular, practical communication channels are commonly affected by packet dropouts and time-delays (for example, due to fading and congestion) [1]. A variety of interesting approaches have been proposed for the analysis and design of NCSs. One class of design methods takes advantage of the fact that in many communication protocols data is sent in large time-stamped packets. This opens the possibility to conceive Sequence-based Control (SBC) formulations, where, through buffering at the receiver, time delays and packet dropouts are to some extent compensated for. Recent works on SBC for network models with bounded packet loss and/or bounded time delays include [2]-[8], which adopt worst-case methods for stability analysis. As shown in the above works, the (somewhat artificial) assumption that network artifacts are bounded is required in order to be able to establish deterministic stability guarantees of SBC. Given the widespread adoption of stochastic models with unbounded support in both the communications and also in the systems control community (see, e.g., [9]-[14]), it is surprising that stochastic models with unbounded support have only recently been taken into account when studying stability of SBC. In particular, [15] investigates quantized control of LTI systems with independent and identically distributed (i.i.d.) dropouts and establishes sufficient condition for mean-square stability of the NCS, whereas [16] focuses on stochastic stability of nonlinear plant models in the presence of Markovian dropouts. The work [17] studies an SBC formulation for LTI systems, where the network introduces possibly unbounded dropouts and delays, under the assumption that acknowledgments of receipt are always available.

In the present work, we study an SBC formulation for discretetime non-linear plant models, where plant states and tentative input sequences are transmitted over an unreliable communication channel, see Fig. 1. As in our previous works [15], [16], the control

Daniel Quevedo is with the School of Electrical Engineering \& Computer Science, The University of Newcastle, NSW 2308, Australia; e-mail: dquevedo@ieee.org. Isabel Jurado is with Departamento de Ingeniería de Sistemas y Automática, Escuela Superior de Ingenieros, Universidad de Sevilla, Spain; e-mail: ijurado@cartuja.us.es. This research was supported under Australian Research Council's Discovery Projects funding scheme (project number DP0988601).

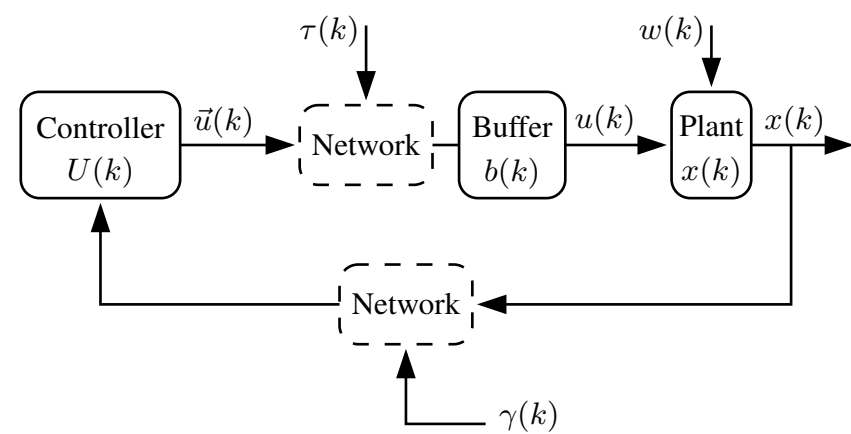

Fig. 1. NCS architecture with buffer at the actuator node

scheme studied does not require acknowledgments of receipts or any knowledge of the packet delay/dropout distributions. Instead, control sequences are designed by applying a pre-designed nominally stabilizing state-feedback control mapping to predicted plant states. Thus, control values are calculated sequentially, reutilizing the already computed values. This is computationally attractive and differs from our previous works [15], [16], which examine the design of packetized optimization-based model predictive controllers. Through the development of a Markovian model of the NCS at hand, we show how our recent results in [18] can be adapted to the present situation and establish sufficient conditions for stochastic stability of the NCS class considered. Our current results are applicable to input-constrained open-loop unstable plants and to networks which introduce i.i.d. time-delays and i.i.d. packet dropouts, in which case the number of consecutive dropouts have a geometric distribution.

Notation: We write $\mathbb{R}_{\geq 0}$ for $[0, \infty), \mathbb{N}$ for $\{1,2, \ldots\}$, and $\mathbb{N}_{0}$ for $\mathbb{N} \cup\{0\}$. The $p \times p$ identity matrix is denoted $I_{p}$. For the all zeroes matrix in $\mathbb{R}^{p \times q}$, we write $0_{p \times q}$. The unit column vector in $\mathbb{R}^{p}$ is $\mathbf{1}_{p}=\left[\begin{array}{llll}1 & 0 & \ldots & 0\end{array}\right]^{T}$, whereas $\mathbf{0}_{p}=0 \cdot \mathbf{1}_{p}$. The norm of a vector $x$ is denoted $|x|$. A function $\varphi: \mathbb{R}_{\geq 0} \rightarrow \mathbb{R}_{\geq 0}$ is of class- $\mathscr{K}_{\infty}$ $\left(\varphi \in \mathscr{K}_{\infty}\right)$, if it is continuous, zero at zero, strictly increasing, and unbounded. $\operatorname{Pr}\{\cdot\}$ refers to probability and $\mathbf{E}\{\cdot\}$, to expectation.

\section{NCS ARCHITECTURE}

We consider (possibly unstable) plant models with state $x \in \mathbb{R}^{n}$ and constrained input $u \in \mathbb{U} \subseteq \mathbb{R}^{p}$, with $\mathbf{0}_{p} \in \mathbb{U}$. The plant state trajectory obeys the recursion:

$$
x(k+1)=f(x(k), u(k)), \quad k \in \mathbb{N}_{0},
$$

where $f\left(\mathbf{0}_{n}, \mathbf{0}_{p}\right)=\mathbf{0}_{n}$. The initial state $x(0)$ is arbitrarily distributed. Throughout this work, we will assume that the nominal plant model (1) is globally stabilizable via state feedback. We adopt an emulation-based approach (cf., [19]), for a pre-designed controller $\kappa$, which is nominally stabilizing in the absence of network effects (see Section VI for a specific example):

Assumption 1: There exist $\kappa: \mathbb{R}^{n} \rightarrow \mathbb{U}, V: \mathbb{R}^{n} \rightarrow \mathbb{R}_{\geq 0}, \varphi_{1}, \varphi_{2} \in$ $\mathscr{K}_{\infty}, \rho \in[0,1)$, such that

$$
\begin{aligned}
\varphi_{1}(|x|) \leq V(x) & \leq \varphi_{2}(|x|), \\
V(f(x, \kappa(x))) & \leq \rho V(x), \quad \forall x \in \mathbb{R}^{n} .
\end{aligned}
$$


1) Network Effects and Buffering: We focus on a situation where transmissions from the sensor to the actuator are over a wireless channel. Due to fading and interference from other users, random dropouts will occur [20]-[23]. For our purposes, their effect is described by introducing the binary transmission success process $\{\gamma(k)\}_{k \in \mathbb{N}_{0}}$, where (see Fig. 1)

$$
\gamma(k)= \begin{cases}1 & \text { if the controller receives } x(k) \text { at time } k, \\ 0 & \text { otherwise. }\end{cases}
$$

The controller calculates control commands $\vec{u}(k)$ only at the times when data arrives, i.e., when $\gamma(k)=1$. Transmissions from controller to actuator use a shared network. Fading, interference and medium access effects introduce random time-delays and dropouts in the controller-actuator link [24]. Here, we introduce $\{\tau(k)\}_{k \in \mathbb{N}_{0}}$, where

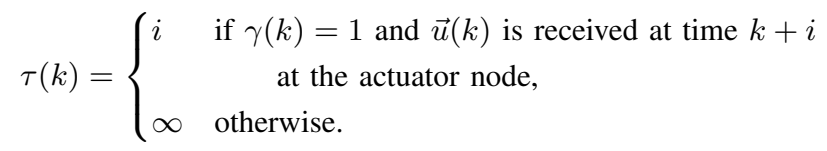

As foreshadowed in the introduction, we are interested in SBC, where $\vec{u}(k)$ contains tentative plant inputs for a finite, and fixed, number of $N$ current and future time steps. To make the predictive nature of the controller explicit, we write

$$
\vec{u}(k)=\left[\begin{array}{c}
u(k ; k) \\
u(k+1 ; k) \\
\vdots \\
u(k+N-1 ; k)
\end{array}\right] \in \mathbb{U}^{N} \subseteq \mathbb{R}^{p N},
$$

where the values $u(k+\ell ; k)$ are tentative controls for time $k+\ell$, calculated at time $k$. At the actuator side, the received packets are buffered, see Fig. 1. The buffer uses the time-stamps to only store (parts of) the most recently generated packets received; older packets are discarded. The most recently generated packet contained in the buffer at time $k$ is $\vec{u}(T(k))$, where

$$
T(k) \triangleq \max \left\{\ell \in \mathbb{N}_{0}: \ell+\tau(\ell) \leq k\right\} .
$$

If $T(k)>k-N$, then the buffer state at time $k$ contains elements of $\vec{u}(T(k))$ as per

$$
b(k)=\left[\begin{array}{c}
u(k ; T(k)) \\
u(k+1 ; T(k)) \\
\vdots \\
u(T(k)+N-1 ; T(k))
\end{array}\right]
$$

and the plant input is set to $u(k)=u(k ; T(k))$. If $T(k) \leq k-N$, then the buffer is empty, $b(k)=\emptyset$, and the plant input is set to zero. ${ }^{1}$ We will assume that the buffer is initially empty:

$$
b(0)=\emptyset, \quad u(0)=\mathbf{0}_{p} .
$$

2) Sequence-Based Controller: Throughout this work, we will focus on the challenging $U D P$-like case where the network provides no acknowledgments of receipt. Therefore, the buffer contents are not available for control calculations. The control sequences $\vec{u}(k)$ in (5) are obtained by applying the mapping $\kappa$ of (2) on predicted nominal plant states, say $x(k+j ; k)$, see (1):

$$
\begin{aligned}
u(k+j ; k) & =\kappa(x(k+j ; k)), \quad j \in\{0,1, \ldots, N-1\}, \\
x(k+j+1 ; k) & =f(x(k+j ; k), u(k+j ; k)), \quad x(k ; k)=x(k) .
\end{aligned}
$$

With SBC, it can be expected that larger horizon lengths $N$ will give better performance, since more delay and dropout scenarios can

\footnotetext{
${ }^{1}$ Alternatively, one could also hold the previous control input; cf., [25].
}

be compensated for. This will become apparent in our subsequent results, in particular, those included in Section VI.

\section{NCS DESIGN MODEL}

Due to the presence of time delays and the buffering procedure adopted, future plant states depend not only upon the current plant state $x(k)$, but also on delay realizations and control packets transmitted after $T(k) \leq k$. To model the situation, we introduce the overall NCS state $\theta(k) \triangleq\left[\begin{array}{ll}x(k)^{T} & U(k)^{T}\end{array}\right]^{T}$, where

$$
\begin{aligned}
U(k) & \triangleq\left[\begin{array}{c}
C_{1} \vec{u}(k-1) \\
C_{2} \vec{u}(k-2) \\
\vdots \\
C_{\bar{N}-1} \vec{u}(k-\bar{N}+1)
\end{array}\right] \in \mathbb{R}^{\nu p}, \\
C_{i} & \triangleq\left[0_{(N-i) p \times i p} \quad I_{(N-i) p}\right], \\
\nu & \triangleq(\bar{N}-1) \bar{N} / 2,
\end{aligned}
$$

with $\bar{N} \triangleq \max \{\Gamma \cap\{0,1, \ldots, N\}\}$ and where $\Gamma \triangleq\{i \in$ $\left.\mathbb{N}_{0}: \operatorname{Pr}\{\tau(k)=i\}>0\right\}$.

Our subsequent analysis makes use of the buffer length process $\{\lambda(k)\}_{k \in \mathbb{N}_{0}}$, defined via,

$$
\lambda(k) \triangleq \begin{cases}\max (0, T(k)+N-k) & \text { if } k \in \mathbb{N} \\ 0 & \text { if } k=0\end{cases}
$$

where $T(k)$ is as in (6). In view of (7), if $\lambda(k)>0$, then we have $b(k) \in \mathbb{U}^{\lambda(k)}$, whereas $\lambda(k)=0$ refers to an empty buffer, $b(k)=\emptyset$. The plant inputs are given by:

$$
u(k)= \begin{cases}u(k ; \lambda(k)+k-N) & \text { if } \lambda(k)>0, \\ \mathbf{0}_{p} & \text { if } \lambda(k)=0 .\end{cases}
$$

Note that $\lambda(k)$ also serves to describe the age of the buffer content; cf., [7].

Expression (12) now gives that, if $\lambda(k)>0$, then $T(k)=\lambda(k)+$ $k-N$, thus

$$
u(k)=u(k ; \lambda(k)+k-N)=G_{\lambda(k)} U(k)+E_{\lambda(k)} \vec{u}(k)
$$

where

$$
\begin{aligned}
G_{\lambda(k)} & =\left\{\begin{array}{ll}
0_{p \times \nu p} & \text { if } \lambda(k)=N, \\
{\left[\begin{array}{lll}
0_{p \times \eta p} & I_{p} & 0_{p \times(\nu-1-\eta) p}
\end{array}\right]} & \text { if } \lambda(k)<N,
\end{array},\right. \\
E_{\lambda(k)} & = \begin{cases}{\left[\begin{array}{ll}
I_{p} & 0_{p \times(N-1) p}
\end{array}\right]} & \text { if } \lambda(k)=N, \\
0_{p \times N p} & \text { if } \lambda(k)<N,\end{cases} \\
\eta & =N-\lambda(k)+(N-\lambda(k)-1) \bar{N} \\
& -(N-\lambda(k))(N-\lambda(k)-1) / 2 .
\end{aligned}
$$

On the other hand, irrespective of $\lambda(k)$, we have

$$
\begin{aligned}
& U(k+1)=S U(k)+\left[\begin{array}{c}
I_{p} \\
0_{(\nu-1) p \times p}
\end{array}\right] \vec{u}(k), \\
& S \triangleq\left[\begin{array}{c}
0_{p \times \nu p} \\
{\left[I_{(\nu-1) p} \quad 0_{(\nu-1) p \times p}\right]}
\end{array}\right] .
\end{aligned}
$$

The above expressions lead to the jump non-linear NCS model:

$$
\begin{aligned}
& \theta(k+1)=F_{\lambda(k)}(\theta(k), \vec{u}(k)), \quad k \in \mathbb{N}_{0}, \\
& F_{\lambda}(\theta, \vec{u}) \triangleq\left[\begin{array}{c}
f\left(x, G_{\lambda} U+E_{\lambda} \vec{u}\right) \\
S U+\left[\begin{array}{c}
I_{p} \\
0_{(\nu p-p) \times p}
\end{array}\right] \vec{u}
\end{array}\right], \quad \theta=\left[\begin{array}{c}
x \\
U
\end{array}\right] .
\end{aligned}
$$

In the following section, we will study a particular instance of the NCS model obtained. Section V then incorporates the control sequences $\vec{u}(k)$, designed as in (9), to study stability of the NCS. 


\section{THE I.I.D. CASE}

In the sequel, we focus on networks, where delays and dropouts are uncorrelated and make the following two assumptions.

Assumption 2: The transmission success process $\{\gamma(k)\}_{k \in \mathbb{N}_{0}}$ is i.i.d., with

$$
\operatorname{Pr}\{\gamma(k)=1\}=q .
$$

The process $\{\tau(k)\}_{k \in \mathbb{N}_{0}}$ is conditionally i.i.d., with delay distribution

$$
\operatorname{Pr}\{\tau(k)=i \mid \gamma(k)=1\}=p_{i}, \quad i \in \mathbb{N}_{0},
$$

and dropout probability $\operatorname{Pr}\{\tau(k)=\infty \mid \gamma(k)=1\}=p_{\infty}$.

A direct consequence of our model is that the unconditional distribution of $\{\tau(k)\}_{k \in \mathbb{N}_{0}}$ satisfies, for all $i \in \mathbb{N}_{0}$,

$$
\begin{gathered}
\operatorname{Pr}\{\tau(k)=i\}=\operatorname{Pr}\{\gamma(k)=1\} \operatorname{Pr}\{\tau(k)=i \mid \gamma(k)=1\}=q p_{i}, \\
\operatorname{Pr}\{\tau(k)=\infty\}=q p_{\infty}+\operatorname{Pr}\{\gamma(k)=0\} \operatorname{Pr}\{\tau(k)=\infty \mid \gamma(k)=0\} \\
=q \cdot p_{\infty}+(1-q)
\end{gathered}
$$

Note that, if $p_{\infty}>0$ or $q<1$, then the number of consecutive packet dropouts in the loop has unbounded support. Thus, even though the controller transmits sequences, with non-zero probability the plant will be left in open loop during intervals of infinite length. This begs the question of stability of this NCS architecture, which we address in Section V.

Remark 1 (Special cases): A particular case of our model results when $q=1$ and $p_{i}=0$ for all $i \in \mathbb{N}$, in which case the controller to actuator network reduces to an erasure channel with dropout rate $p_{\infty}=1-p_{0}$. The latter model was adopted for examining SBC over bit-rate limited channels in [15]. Another instance studied in previous literature is where $q=1$ and time-delays have a bounded support, i.e., there exists $\tau^{\max } \in \mathbb{N}_{0}$, such that

$$
p_{i}=0, \quad \forall i>\tau^{\max }, i \neq \infty,
$$

in which case the dropout rate is given by

$$
p_{\infty}=1-\sum_{i=0}^{\tau^{\max }} p_{i}
$$

For the special case of (15) with no dropouts, we have $\sum_{i=0}^{\tau^{\max }} p_{i}=1$ as considered, for example, in [2], [8], [26].

A key property of the NCS model obtained is that, for delays and dropouts satisfying Assumption 2, the process $\{\lambda(k)\}_{k \in \mathbb{N}_{0}}$ introduced in (11) constitutes a homogeneous (finite) Markov Chain. Its transition probabilities

$$
p_{i j} \triangleq \operatorname{Pr}\{\lambda(k+1)=j \mid \lambda(k)=i\}
$$

are as follows: ${ }^{2}$

Lemma 1: If Assumption 2 holds, then the transition probabilities of $\{\lambda(k)\}_{k \in \mathbb{N}_{0}}$ satisfy:

$$
p_{i j}= \begin{cases}q \cdot p_{N-j} & \text { if } 1 \leq i \leq j \\ 1-q \sum_{\ell=0}^{N-i} p_{\ell} & \text { if } i=j+1 \\ 0 & \text { if } i \geq j+2 \\ p_{1 j} & \text { if } i=0\end{cases}
$$

where $i, j \in\{0,1, \ldots, N\}$.

Proof: See Appendix A.

${ }^{2} \mathrm{~A}$ related characterization can also be found in parallel work documented in [17].

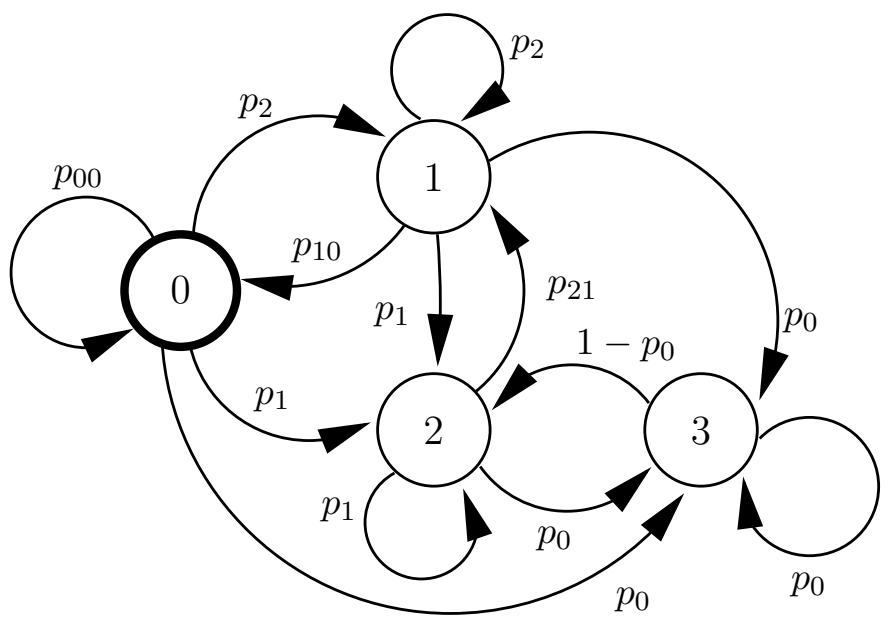

Fig. 2. Transition graph of $\{\lambda(k)\}_{k \in \mathbb{N}}$ in (17). Here, $p_{10}=p_{00}=1-$ $p_{0}-p_{1}-p_{2}$ and $p_{21}=1-p_{0}-p_{1}$.

Example 1: With horizon length $N=3$ and $q=1$, expression (16) yields the transition matrix

$$
\left[p_{i j}\right]=\left[\begin{array}{cccc}
1-p_{0}-p_{1}-p_{2} & p_{2} & p_{1} & p_{0} \\
1-p_{0}-p_{1}-p_{2} & p_{2} & p_{1} & p_{0} \\
0 & 1-p_{0}-p_{1} & p_{1} & p_{0} \\
0 & 0 & 1-p_{0} & p_{0}
\end{array}\right]
$$

Fig. 2 illustrates the associated transition graph.

We will next adapt ideas, which were used in [18] for the analysis of control loops without time-delays or dropouts, but where processor availability is random. Our analysis differentiates between time steps where $\lambda(k)=0$ and, thus, the buffer is empty, and those time instances where the buffer contains plant inputs which were calculated by the controller. For future reference, we denote the times when $b(k)=\emptyset$ via $\mathbb{K}=\left\{k_{i}\right\}_{i \in \mathbb{N}_{0}}$, where $k_{0}=0$, see (8), and

$$
k_{i+1}=\inf \left\{k \in \mathbb{N}: k>k_{i}, \lambda(k)=0\right\}, \quad i \in \mathbb{N}_{0} .
$$

We also introduce the process $\left\{\Delta_{i}\right\}_{i \in \mathbb{N}_{0}}$, where

$$
\Delta_{i} \triangleq k_{i+1}-k_{i}, \quad \forall\left(k_{i+1}, k_{i}\right) \in \mathbb{K} \times \mathbb{K}
$$

are the number of time steps between consecutive instants in $\mathbb{K}$. This process thereby amounts to the first return times of $\{\lambda(k)\}_{k \in \mathbb{N}_{0}}$ to state 0 and is i.i.d. [27]; see also Fig. 2. The distribution of $\left\{\Delta_{i}\right\}_{i \in \mathbb{N}_{0}}$ can be characterised as follows:

Lemma 2: Suppose that Assumption 2 holds and define

$\sigma^{T} \triangleq q\left[\begin{array}{llll}p_{N-1} & \cdots & p_{1} & p_{0}\end{array}\right], \mathcal{P} \triangleq\left[\begin{array}{cccc}p_{11} & p_{12} & \ldots & p_{1 N} \\ \vdots & \vdots & & \vdots \\ p_{N 1} & p_{N 2} & \cdots & p_{N N}\end{array}\right]$,

with $p_{i j}$ as in (16). Then

$$
\operatorname{Pr}\left\{\Delta_{i}=j\right\}= \begin{cases}1-q \sum_{\ell=0}^{N-1} p_{\ell} & \text { if } j=1 \\ \left(1-q \sum_{\ell=0}^{N-1} p_{\ell}\right) \sigma^{T} \mathcal{P}^{j-2} \mathbf{1}_{N} & \text { if } j \geq 2\end{cases}
$$

Proof: See Appendix B.

It is intuitively clear that, to achieve good control performance, $\Delta_{i}$ should be "large". This observation will be confirmed by the results established in the following section.

\section{StABILITy ANALYSis}

Due to the occurrence of random time-delays and dropouts, the plant input is also random. In particular, at all instants where $\lambda(k)<$ 


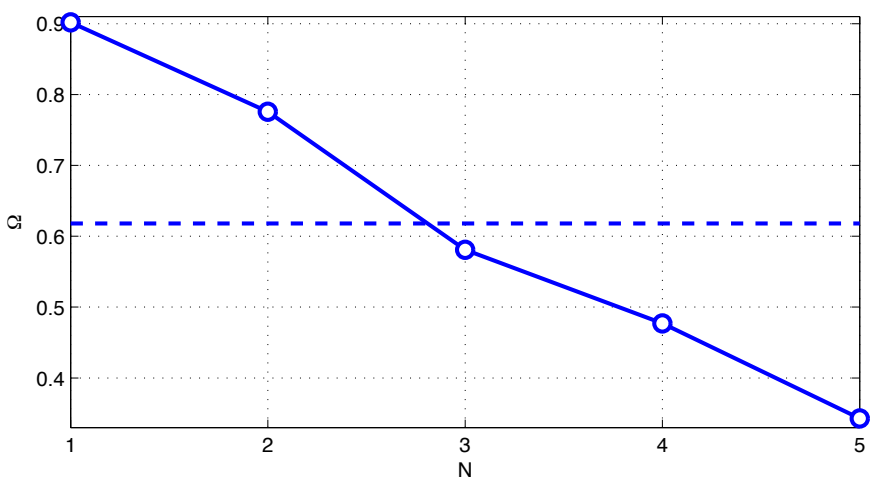

Fig. 3. $\Omega$ as a function of $N$. The horizontal line depicts the stability condition, $\Omega \leq 1 / \alpha \approx 0.618$.

$N$, the plant is unavoidably left in open-loop; if $\lambda(k)=0$, then $u(k)=\mathbf{0}_{p}$. This observation motivates us to study stochastic stability of the NCS model.

Definition 1 (Stochastic Stability): The NCS is stochastically stable, if for some $\varphi \in \mathscr{K}_{\infty}$, the plant state trajectory $\{x(k)\}_{k \in \mathbb{N}_{0}}$ satisfies $\sum_{k=0}^{\infty} \mathbf{E}\{\varphi(|x(k)|)\}<\infty$.

Assumption 3 bounds the rate of increase of $V$ in (2), when the plant input is zero. It also imposes a restriction on the distribution of the initial plant state, see Section VI for an example.

Assumption 3: There exists $\alpha \in \mathbb{R}_{\geq 0}$ such that

$$
V\left(f\left(x, \mathbf{0}_{p}\right)\right) \leq \alpha V(x), \quad \forall x \in \mathbb{R}^{n},
$$

and $\mathbf{E}\left\{\varphi_{2}(|x(0)|)\right\}<\infty$, where $V$ and $\varphi_{2}$ are as in (2). ${ }^{3}$

The results of Section IV allow one to adopt a stochastic Lyapunov function approach to study stability of the NCS described by (1), (9) and (12). We begin by stating the following lemma:

Lemma 3: Suppose that Assumptions 1 to 3 hold and consider $k_{0}, k_{1} \in \mathbb{K}$, see (18). Then

$$
\begin{aligned}
& \mathbf{E}\left\{V\left(x\left(k_{1}\right)\right) \mid \theta\left(k_{0}\right)=\vartheta\right\} \leq \alpha \sum_{j \in \mathbb{N}} \operatorname{Pr}\left\{\Delta_{i}=j\right\} \rho^{j-1} V(\chi), \\
& \forall \vartheta=\left[\begin{array}{c}
\chi \\
\mathcal{U}
\end{array}\right] \in \mathbb{R}^{n+\nu p},
\end{aligned}
$$

where the distribution of $\left\{\Delta_{i}\right\}_{i \in \mathbb{N}_{0}}$ is given in (20).

Proof: See Appendix C.

Whilst, in general, $\{\theta(k)\}_{k \in \mathbb{N}_{0}}$ is not Markovian, $\left\{\theta\left(k_{i}\right)\right\}_{k_{i} \in \mathbb{K}}$ is Markovian and we can use Lemma 3 to derive the following sufficient condition for stochastic stability of the NCS:

Theorem 1: Suppose that Assumptions 1 to 3 hold and define

$$
\Omega \triangleq\left(1-q \sum_{\ell=0}^{N-1} p_{\ell}\right)\left(1+\rho \sigma^{T} \sum_{j \in \mathbb{N}_{0}}(\rho \mathcal{P})^{j} \mathbf{1}_{N}\right)<\infty,
$$

see (19). If $\Omega<1 / \alpha$, then the NCS described by (1), (9) and (12) is stochastically stable.

Proof: See Appendix D.

The above result establishes conditions on the network, plant and controller, which guarantee that the closed loop in the presence of dropouts and delays will be stochastically stable. It is worth noting that with SBC, control packets which are delayed longer than $N-1$ time-steps are discarded. This is reflected in (22) by the fact that $\Omega$ depends on $p_{0}, \ldots, p_{N-1}, q$ and $\rho$ only. We also emphasize that the condition $\Omega \alpha<1$ does not require contractiveness for each step $k \in$

${ }^{3}$ If $\alpha$ cannot be found for given $V$, then the current results are noninformative and the question of controller-redesign arises.

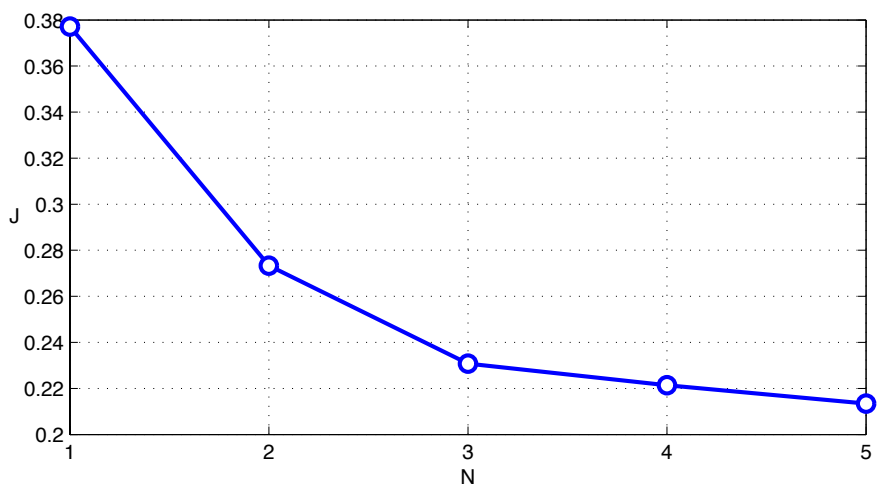

Fig. 4. Empirical expectation of $J \triangleq(1 / 50) \sum_{k=0}^{49}|x(k)|^{2}$, as a function of the buffer length $N$.

$\mathbb{N}$. Instead, it only amounts contractiveness of the process sampled at $k \in \mathbb{K}$.

Remark 2 (Disturbances): The present work focuses on controlling a disturbance-free system model of the form (1). Our current results can be extended to encompass non-zero disturbances, provided that continuity assumptions are imposed on the plant model and the feedback law $\kappa$. Such an analysis was carried out for a related problem in our recent paper [16]. By constraining sample-path differences between the nominal and the perturbed model to be close in a sample path sense, [16] established the boundedness of moments for the plant state process. Such an approach can be adopted for the present control formulation as well.

\section{Simulation StUdY}

Consider an open-loop unstable constrained plant model of the form (1), where

$$
f(x, u)=\left[\begin{array}{c}
x_{2}+u_{1} \\
-\operatorname{sat}\left(x_{1}+x_{2}\right)+u_{2}
\end{array}\right]
$$

with

$$
x=\left[\begin{array}{l}
x_{1} \\
x_{2}
\end{array}\right], u=\left[\begin{array}{l}
u_{1} \\
u_{2}
\end{array}\right], \quad \operatorname{sat}(\mu)= \begin{cases}-1, & \text { if } \mu<-1, \\
\mu & \text { if } \mu \in[-1,1], \\
1, & \text { if } \mu>1,\end{cases}
$$

see [28, Example 2.3] and [16]. The initial state is taken from a Gaussian distribution with zero mean and variance $I_{2}$. We fix $|\cdot|$ as the Euclidean norm. The second component of the plant input is constrained via $\left|u_{2}(k)\right| \leq 0.8, \forall k \in \mathbb{N}_{0}$, thus, $\mathbb{U}=\mathbb{R} \times[-0.8,0.8]$.

The model (23) can be globally stabilized (in the absence of network effects) by the constrained control law $\kappa: \mathbb{R}^{2} \rightarrow \mathbb{U}$, where

$$
\kappa(x)=\left[\begin{array}{ll}
-x_{2} & 0.8 \operatorname{sat}\left(x_{1}+x_{2}\right)
\end{array}\right]^{T} .
$$

In fact, with $V(x)=2|x|$, direct calculations give

$$
\begin{aligned}
& V(f(x, \kappa(x)))=0.4\left|\operatorname{sat}\left(x_{1}+x_{2}\right)\right| \leq 0.4\left|x_{1}+x_{2}\right| \\
& \leq 0.8 \max \left\{\left|x_{1}\right|,\left|x_{2}\right|\right\}-\max \left\{\left|x_{1}\right|,\left|x_{2}\right|\right\}+|x| \leq|x| .
\end{aligned}
$$

Thus, Assumption 1 holds with $\rho=1 / 2$, and $\varphi_{1}(s)=\varphi_{2}(s)=2 s$. Furthermore, by proceeding as in [28, p.73], it can be shown that (21) holds with $\alpha=1.618$.

The network introduces i.i.d. delays and dropouts satisfying Assumption 2, with $q=0.9, p_{0}=0.2, p_{1}=p_{2}=0.25, p_{3}=p_{4}=$ $0.1, p_{5}=p_{\infty}=0.05$. Fig. 3 depicts the associated quantity $\Omega$, see (22), as a function of the horizon length (buffer size) $N$. By recalling Theorem 1 , the figure illustrates that using larger horizons is beneficial for guaranteeing stability. In particular, the NCS is stochastically stable for horizon lengths $N \geq 3$. 
Fig. 4 compares the empirical expectation of $|x(k)|^{2}$ as a function of $N$; results are averaged out over 100 realizations of the initial state and transmission outcome realizations. It can be seen that performance improves monotonically with the buffer length.

\section{CONCLUSIONS}

We have studied a control formulation for non-linear constrained plant models where communications between controller and plant are affected by random dropouts and delays. To compensate for transmission effects, the controller transmits sequences of tentative plant inputs to a buffer at the actuator node. By developing a NCS model which makes the size of the buffer contents explicit, we have established sufficient conditions for stochastic stability when dropouts and delays are i.i.d. Future work of interest includes studying temporally and spatially correlated time-delays and dropouts. For that purpose, we foresee that the models developed in [22], [23] could serve as a starting point.

\section{REFERENCES}

[1] J. Chen, K. H. Johansson, S. Olariu, I. C. Paschalidis, and Stokmenovic, "Guest editorial special issue on wireless sensor and actuator networks," IEEE Trans. Automat. Contr., vol. 56, pp. 2244-2246, Oct. 2011.

[2] P. L. Tang and C. W. de Silva, "Stability validation of a constrained model predictive networked control system with future input buffering," Int. J. Contr., vol. 80, pp. 1954-1970, Dec. 2007.

[3] I. G. Polushin, P. X. Liu, and C.-H. Lung, "On the model-based approach to nonlinear networked control systems," Automatica, vol. 44, pp. 24092414, 2008.

[4] D. Muñoz de la Peña and P. D. Christofides, "Lyapunov-based model predictive control of nonlinear systems subject to data losses," IEEE Trans. Automat. Contr., vol. 53, pp. 2076-2089, Sept. 2008.

[5] J. Liu, D. Muñoz de la Peña, P. D. Christofides, and J. F. Davis, "Lyapunov-based model predictive control of nonlinear systems subject to time-varying measurement delays," Int. J. Adapt. Contr. Signal Proc., vol. 23, pp. 788-807, 2009.

[6] R. Wang, G.-P. Liu, W. Wang, D. Rees, and Y.-B. Zhao, " $H_{\infty}$ control for networked predictive control systems based on the switched Lyapunov function method," IEEE Trans. Ind. Electron., vol. 57, pp. 3565-3571, Oct. 2010

[7] G. Pin and T. Parisini, "Networked predictive control of uncertain constrained nonlinear systems: Recursive feasibility and input-to-state stability analysis," IEEE Trans. Automat. Contr., vol. 56, pp. 72-87, Jan. 2011.

[8] D. E. Quevedo and D. Nešić, "Input-to-state stability of packetized predictive control over unreliable networks affected by packet-dropouts," IEEE Trans. Automat. Contr., vol. 56, pp. 370-375, Feb. 2011.

[9] A. Goldsmith, Wireless Communications. Cambridge University Press, 2005.

[10] R. A. Berry and R. G. Gallager, "Communication over fading channels with delay constraints," IEEE Trans. Inform. Theory, vol. 48, pp. 11351149, May 2002.

[11] L. Schenato, "Optimal estimation in networked control systems subject to random delay and packet drop," IEEE Trans. Automat. Contr., vol. 53, pp. 1311-1317, June 2008.

[12] L. Shi, "Kalman filtering over graphs: Theory and applications," IEEE Trans. Automat. Contr., vol. 54, pp. 2230-2234, Sept. 2009.

[13] Q. Ling and M. D. Lemmon, "Power spectral analysis of networked control systems with data dropouts," IEEE Trans. Automat. Contr. vol. 49, pp. 955-960, June 2004

[14] V. Gupta, B. Hassibi, and R. M. Murray, "Optimal LQG control across packet-dropping links," Syst. \& Contr. Lett., vol. 56, pp. 439-446, 2007.

[15] D. E. Quevedo, J. Østergaard, and D. Nešić, "Packetized predictive control of stochastic systems over bit-rate limited channels with packet loss," IEEE Trans. Automat. Contr., vol. 56, pp. 2854-2868, Dec. 2011.

[16] D. E. Quevedo and D. Nešić, "Robust stability of packetized predictive control of nonlinear systems with disturbances and Markovian packet losses," Automatica, vol. 48, pp. 1803-1811, Aug. 2012.

[17] J. Fischer, M. Dolgov, and U. Hanebeck, "On stability of sequence-based LQG control," in Proc. IEEE Conf. Decis. Contr., 2013.

[18] D. E. Quevedo and V. Gupta, "Stability of sequence-based anytime control with Markovian processor availability," in Proc. Austr. Contr. Conf., 2011.
[19] W. P. M. H. Heemels, A. R. Teel, N. van de Wouw, and D. Nešić, "Networked control systems with communication constraints: tradeoffs between transmission intervals, delays and performance," IEEE Trans. Automat. Contr., vol. 55, no. 8, pp. 1781-1796, 2010.

[20] D. E. Quevedo, A. Ahlén, and J. Østergaard, "Energy efficient state estimation with wireless sensors through the use of predictive power control and coding," IEEE Trans. Signal Processing, vol. 58, pp. 48114823, Sept. 2010.

[21] L. Schenato, B. Sinopoli, M. Franceschetti, K. Poolla, and S. S. Sastry, "Foundations of control and estimation over lossy networks," Proc. IEEE, vol. 95, pp. 163-187, Jan. 2007.

[22] D. E. Quevedo, A. Ahlén, A. S. Leong, and S. Dey, "On Kalman filtering over fading wireless channels with controlled transmission powers," Automatica, vol. 48, pp. 1306-1316, July 2012.

[23] D. E. Quevedo, A. Ahlén, and K. H. Johansson, "State estimation over sensor networks with correlated wireless fading channels," IEEE Trans. Automat. Contr., vol. 58, pp. 581-593, Mar. 2013.

[24] C. Ramesh, H. Sandberg, and K. H. Johansson, "Steady state performance analysis of multiple state-based schedulers with CSMA," in Proc. IEEE Conf. Decis. Contr., 2011.

[25] L. Schenato, "To zero or to hold control inputs with lossy links?," IEEE Trans. Automat. Contr., vol. 54, pp. 1093-1099, May 2009.

[26] G. P. Liu, J. X. Mu, D. Rees, and S. C. Chai, "Design and stability analysis of networked control systems with random communication time delay using the modified MPC," Int. J. Contr., vol. 79, pp. 288-297, Apr. 2006.

[27] J. G. Kemeny and J. L. Snell, Finite Markov Chains. D. Van Nostrand Company, Inc, 1960.

[28] H. K. Khalil, Nonlinear Systems. Prentice Hall, 2nd ed., 1996.

[29] H. Kushner, Introduction to Stochastic Control. New York, N.Y.: Holt, Rinehart and Winston, Inc., 1971.

\section{APPENDIX}

\section{A. Proof of Lemma 1}

We first note that transitions corresponding to $i \geq j+2$ will never occur, since the buffer can only loose, at most, one control value in one transition, thus, $p_{i j}=0$, for $i \geq j+2$.

For the cases $1 \leq i \leq j$, the buffer is never empty and some control sequence generated after $T(k)$ is received at instant $k+1$. Since $\lambda(k)=N-\ell>0$ if and only if $\tau(k-\ell) \leq \ell$ and $\tau(k-\ell+i)>\ell-i$ for all $i \in\{1,2, \ldots, \ell\}$, a careful case-by-case analysis considering all the possibilities and taking into account that the size of the buffer is $N$ reveals that

$$
\begin{aligned}
& p_{i j}=\operatorname{Pr}\{\lambda(k+1)=j \mid \lambda(k)=i\} \\
& =\operatorname{Pr}\{\tau(j-N+k+1) \leq N-j \\
& \quad \mid \tau(j-N+k+1)>N-j-1\} \\
& \quad \times \prod_{\ell=2}^{N+1-j} \operatorname{Pr}\{\tau(j-N+k+\ell)>N-j-\ell+1 \\
& \quad \mid \tau(j-N+k+\ell)>N-j-\ell\} \\
& =\frac{\operatorname{Pr}\{\tau(j-N+k+1)=N-j\}}{\operatorname{Pr}\{\tau(j-N+k+1)>N-j-1\}} \\
& \prod_{\ell+1-j}^{N-1} \frac{\operatorname{Pr}\{\tau(j-N+k+\ell)>N-j-\ell+1\}}{\operatorname{Pr}\{\tau(j-N+k+\ell)>N-j-\ell\}}=q \cdot p_{N-j}
\end{aligned}
$$

since $\{\tau(k)\}_{k \in \mathbb{N}_{0}}$ is i.i.d.

The case $j=i-1$ refers to the event that no "fresh" control sequence arrives to the buffer at the instant $k+1$. The associated 
transition probabilities satisfy:

$$
\begin{aligned}
p_{i j}= & \operatorname{Pr}\{\lambda(k+1)=j \mid \lambda(k)=i\} \\
= & \operatorname{Pr}\{\tau(k-N+1+j) \leq-N+2+j \\
& \mid \tau(k-N+1+j) \leq-N+1+j\} \\
& \times \prod_{\ell=0}^{N-1-j} \operatorname{Pr}\{\tau(k+1-\ell)>\ell \mid \tau(k+1-\ell)>\ell-1\} \\
= & \prod_{\ell=0}^{N-1-j} \frac{\operatorname{Pr}\{\tau(k+1-\ell)>\ell \wedge \tau(k+1-\ell)>\ell-1\}}{\operatorname{Pr}\{\tau(k+1-\ell)>\ell-1\}} \\
= & \prod_{\ell=0}^{N-1-j} \frac{\operatorname{Pr}\{\tau(k+1-\ell)>\ell\}}{\operatorname{Pr}\{\tau(k+1-\ell)>\ell-1\}} \\
= & \prod_{\ell=0}^{N-1-j} \frac{1-q \sum_{m=0}^{\ell} p_{m}}{1-q \sum_{m=0}^{\ell-1} p_{m}}=1-q \sum_{\ell=0}^{N-1-j} p_{\ell}=1-q \sum_{\ell=0}^{N-i} p_{\ell} .
\end{aligned}
$$

Finally, for the case $i=0$, we consider two different events, namely, $j=0$ and $j>0$. Direct calculations give that

$$
\begin{aligned}
p_{00} & =\operatorname{Pr}\{\lambda(k+1)=0 \mid \lambda(k)=0\} \\
& =\prod_{\ell=0}^{N-1} \operatorname{Pr}\{\tau(k+1-\ell)>\ell \mid \tau(k+1-\ell)>\ell-1\} \\
& =\prod_{\ell=0}^{N-1} \frac{\operatorname{Pr}\{\tau(k+1-\ell)>\ell\}}{\operatorname{Pr}\{\tau(k+1-\ell)>\ell-1\}}=p_{10} .
\end{aligned}
$$

On the other hand, for $j>0$, we obtain

$$
\begin{aligned}
p_{0 j}= & \operatorname{Pr}\{\lambda(k+1)=j \mid \lambda(k)=0\} \\
= & \frac{\operatorname{Pr}\{\tau(j-N+k+1)=N-j\}}{\operatorname{Pr}\{\tau(j-N+k+1)>N-j-1\}} \\
& \times \prod_{\ell=2}^{N+1-j} \frac{\operatorname{Pr}\{\tau(j-N+k+\ell)>N-j-\ell+1\}}{\operatorname{Pr}\{\tau(j-N+k+\ell)>N-j-\ell\}}=p_{1 j},
\end{aligned}
$$

which proves the result.

\section{B. Proof of Lemma 2}

The proof is akin to that of Lemma 2 of [18]. The case $j=1$ is immediate, since

$$
\operatorname{Pr}\left\{\Delta_{i}=1\right\}=\operatorname{Pr}\{\lambda(k+1)=0 \mid \lambda(k)=0\}=p_{00},
$$

see (16). For $j \geq 2$, we analyze the state trajectory of the Markov Chain $\{\lambda(k)\}_{k \in \mathbb{N}_{0}}$ and proceed as follows: For all $m \in\{1, \ldots, N\}$, denote by $\delta_{m}$ the first passage time of the state $m$ to state 0 . Thus, $\delta_{m}$ are discrete random variables, where $\delta_{m}=j$, if $\{\lambda(k)\}_{k \in \mathbb{N}_{0}}$ enters the state 0 from $m$ for the first time in exactly $j$ steps. Since, with exception of state 0 , only the state 1 can reach 0 in one step, it is easy to see that

$$
\operatorname{Pr}\left\{\delta_{m}=1\right\}=p_{m 0}= \begin{cases}1-q \sum_{\ell=0}^{N-1} p_{\ell} & \text { if } m=1 \\ 0 & \text { if } m>1,\end{cases}
$$

where we have used (16). For $j \geq 2$, paths from $m \neq 0$ to 0 go through intermediate states $\ell \neq 0$, providing the recursions

$$
\operatorname{Pr}\left\{\delta_{m}=j\right\}=\sum_{\ell=1}^{N} p_{m \ell} \operatorname{Pr}\left\{\delta_{\ell}=j-1\right\}, \forall m .
$$

Therefore,

$$
\left[\begin{array}{c}
\operatorname{Pr}\left\{\delta_{1}=j\right\} \\
\vdots \\
\operatorname{Pr}\left\{\delta_{N}=j\right\}
\end{array}\right]=\mathcal{P}\left[\begin{array}{c}
\operatorname{Pr}\left\{\delta_{1}=j-1\right\} \\
\vdots \\
\operatorname{Pr}\left\{\delta_{N}=j-1\right\}
\end{array}\right], \quad \forall j \geq 2
$$

yielding the explicit formula:

$$
\left[\begin{array}{c}
\operatorname{Pr}\left\{\delta_{1}=j\right\} \\
\vdots \\
\operatorname{Pr}\left\{\delta_{N}=j\right\}
\end{array}\right]=\mathcal{P}^{j-1}\left[\begin{array}{c}
\operatorname{Pr}\left\{\delta_{1}=1\right\} \\
\vdots \\
\operatorname{Pr}\left\{\delta_{N}=1\right\}
\end{array}\right]=\left(1-q \sum_{\ell=0}^{N-1} p_{\ell}\right) \mathcal{P}^{j-1} \mathbf{1}_{N},
$$

which by virtue of (24) holds, not only for $j \geq 2$, but also for $j=1$. Thus, the distribution of $\left\{\Delta_{i}\right\}$ can be obtained from those of $\left\{\delta_{m}\right\}$ by considering the transitions away from 0 :

$$
\begin{aligned}
\operatorname{Pr}\left\{\Delta_{i}=j\right\} & =\sum_{\ell=1}^{N} p_{0 \ell} \operatorname{Pr}\left\{\delta_{\ell}=j-1\right\}=\sigma^{T}\left[\begin{array}{c}
\operatorname{Pr}\left\{\delta_{1}=j-1\right\} \\
\vdots \\
\operatorname{Pr}\left\{\delta_{N}=j-1\right\}
\end{array}\right] \\
& =\left(1-q \sum_{\ell=0}^{N-1} p_{\ell}\right) \sigma^{T} \mathcal{P}^{j-2} \mathbf{1}_{N},
\end{aligned}
$$

for all $j \geq 2$, where we have used (16) and (25).

\section{Proof of Lemma 3}

Assumption 3 gives that

$$
V\left(x\left(k_{0}+1\right)\right) \leq \alpha V\left(x\left(k_{0}\right)\right), \quad \forall x\left(k_{0}\right) \in \mathbb{R}^{n} .
$$

On the other hand, if $k \notin \mathbb{K}$, then $\lambda(k)>0$ and the plant input stems from current or previously calculated control sequences. Since the control input sequences are constructed following (9), in the disturbance-free case considered, $x(k)=x(k ; T(k))$, where $T(k)$ is as in (6). Therefore, (2) gives that $V(x(k+1)) \leq \rho V(x(k))$, $\forall k \notin \mathbb{K}$. Thus,

$$
\begin{gathered}
\mathbf{E}\left\{V\left(x\left(k_{1}\right)\right) \mid \theta\left(k_{0}\right)=\vartheta, \Delta_{0}=j\right\} \leq \rho^{j-1} \alpha V(\chi), \\
\forall \vartheta=\left[\begin{array}{c}
\chi \\
\mathcal{U}
\end{array}\right] \in \mathbb{R}^{n+\nu p} .
\end{gathered}
$$

Since $\left\{\Delta_{i}\right\}_{i \in \mathbb{N}_{0}}$ is i.i.d., the result follows directly from the law of total expectation.

\section{Proof of Theorem 1}

We first note that

$$
\Omega=\sum_{j \in \mathbb{N}} \operatorname{Pr}\left\{\Delta_{i}=j\right\} \rho^{j-1} .
$$

Since $\rho \in[0,1)$, we have $\Omega<\infty$. Furthermore, $\left\{\theta\left(k_{i}\right)\right\}_{k_{i} \in \mathbb{K}}$ is Markovian. Thus, Lemma 3 and [29, Chapter 8.4.2, Theorem 2] yield exponential stability at instants $k_{i} \in \mathcal{K}$, i.e.,

$$
\mathbf{E}\left\{V\left(x\left(k_{i}\right)\right) \mid \theta\left(k_{0}\right)=\vartheta\right\} \leq(\alpha \Omega)^{i} V(\chi), \quad \forall \vartheta=\left[\begin{array}{c}
\chi \\
\mathcal{U}
\end{array}\right] \in \mathbb{R}^{n+\nu p} .
$$

For the time instants $k \in \mathbb{N} \backslash \mathbb{K}$, i.e., where $\lambda(k)>0$, the plant inputs stem from previously calculated control sequences. Thus, we can use (2) and (26) to bound

$$
\begin{aligned}
& \mathbf{E}\left\{\sum_{k=k_{i}}^{k_{i+1}-1} V(x(k)) \mid x\left(k_{i}\right)=\chi_{i}, \Delta_{i}=j\right\} \leq\left(1+\alpha \sum_{l=0}^{j-2} \rho^{l}\right) V\left(\chi_{i}\right) \\
& \quad \leq\left(1+\frac{\alpha}{1-\rho}\right) V\left(\chi_{i}\right),
\end{aligned}
$$

so that, by the law of total expectation,

$\mathbf{E}\left\{\sum_{k=k_{i}}^{k_{i+1}-1} V(x(k)) \mid \theta\left(k_{i}\right)=\vartheta_{i}\right\} \leq \frac{1+\alpha-\rho}{1-\rho} V\left(\chi_{i}\right), \quad \forall \vartheta_{i}=\left[\begin{array}{c}\chi_{i} \\ \mathcal{U}_{i}\end{array}\right]$.

The result follows from taking conditional expectation $\mathbf{E}\left\{\cdot \mid \theta\left(k_{0}\right)\right\}$, use of the Markovian property of $\left\{\theta\left(k_{i}\right)\right\}_{k_{i} \in \mathbb{K}}$, and proceeding, mutatis mutandis, as in the proof of [18, Thm.1]. 